\title{
The brain transcriptome of the wolf spider, Schizocosa ocreata
}

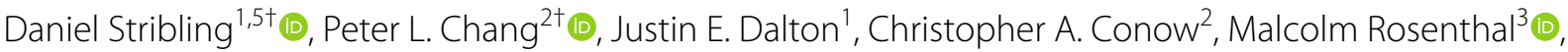 \\ Eileen Hebets ${ }^{3}$, Rita M. Graze ${ }^{4}$ and Michelle N. Arbeitman ${ }^{1 *}$ (D)
}

\begin{abstract}
Objectives: Arachnids have fascinating and unique biology, particularly for questions on sex differences and behavior, creating the potential for development of powerful emerging models in this group. Recent advances in genomic techniques have paved the way for a significant increase in the breadth of genomic studies in non-model organisms. One growing area of research is comparative transcriptomics. When phylogenetic relationships to model organisms are known, comparative genomic studies provide context for analysis of homologous genes and pathways. The goal of this study was to lay the groundwork for comparative transcriptomics of sex differences in the brain of wolf spiders, a non-model organism of the pyhlum Euarthropoda, by generating transcriptomes and analyzing gene expression.

Data description: To examine sex-differential gene expression, short read transcript sequencing and de novo transcriptome assembly were performed. Messenger RNA was isolated from brain tissue of male and female subadult and mature wolf spiders (Schizocosa ocreata). The raw data consist of sequences for the two different life stages in each sex. Computational analyses on these data include de novo transcriptome assembly and differential expression analyses. Sample-specific and combined transcriptomes, gene annotations, and differential expression results are described in this data note and are available from publicly-available databases.
\end{abstract}

Keywords: Schizocosa ocreata, Wolf spiders, Gene expression, Transcriptomes, Sexual dimorphism, Sex-differential gene expression, Sex biased expression, Brain and central nervous system, De novo transcriptome assembly

\section{Objectives}

Arachnids, including spiders, have diverse and unique reproductive behavior, including sexual cannibalism and female aggression, copulatory wounding, and elaborate courtship with sexual dimorphism in morphology and coloration [1-5]. The development of genomic resources in arachnids will allow for key comparisons not only in genome biology, but also in evolution and in the biology of sex. Comparative studies between arachnids and model organisms in other arthropod classes can provide

*Correspondence: michelle.arbeitman@med.fsu.edu

${ }^{\dagger}$ Daniel Stribling and Peter L. Chang contributed equally to this work

${ }^{1}$ Biomedical Sciences Department, College of Medicine, Florida State

University, Tallahassee, FL 32306, USA

Full list of author information is available at the end of the article a broader set of inferences that goes beyond what has been learned from model organisms. For example, copulatory wounding, male leg ornamentation, and elaborate courtship are well-studied in Drosophila, and arachnid genomic comparative studies can reveal parallel or divergent mechanisms [6-15].

Several arachnid genomes and transcriptomes, including those of spiders, mites and scorpions, have recently become available [16-18]. Given that spiders have unique sex-specific behaviors and that progress is ongoing in developing arachnid genomics, our goal was to generate transcriptomes and gene expression data using mRNA from brains of males and females of the wolf spider, Schizocosa ocreata. Studies of wolf spider sexual dimorphism in morphology and behavior have revealed intriguing parallels to textbook examples of sex dimorphism

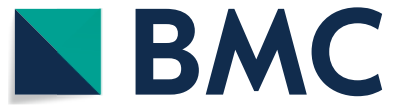

(c) The Author(s) 2021. This article is licensed under a Creative Commons Attribution 4.0 International License, which permits use, sharing, adaptation, distribution and reproduction in any medium or format, as long as you give appropriate credit to the original author(s) and the source, provide a link to the Creative Commons licence, and indicate if changes were made. The images or other third party material in this article are included in the article's Creative Commons licence, unless indicated otherwise in a credit line to the material. If material is not included in the article's Creative Commons licence and your intended use is not permitted by statutory regulation or exceeds the permitted use, you will need to obtain permission directly from the copyright holder. To view a copy of this licence, visit http://creativeco mmons.org/licenses/by/4.0/. The Creative Commons Public Domain Dedication waiver (http://creativecommons.org/publicdomain/ zero/1.0/) applies to the data made available in this article, unless otherwise stated in a credit line to the data. 
Table 1 Overview of data files/data sets

\begin{tabular}{|c|c|c|c|}
\hline Label & Name of data file/data set & File types (file extension) & $\begin{array}{l}\text { Data repository and identifier (DOI or accession } \\
\text { number) }\end{array}$ \\
\hline Data set 1 & Raw illumina data & FASTQ files (.fq) & $\begin{array}{l}\text { NCBI SRA: https://identifiers.org/ncbi/insdc.sra:SRP30 } \\
2932 \text { [45] }\end{array}$ \\
\hline Data file 1 & Trimmed-read FastQC statistics & PDF file (.pdf) & NCBI GEO: https://identifiers.org/geo:GSE168766 [46] \\
\hline Data set 2 & $\begin{array}{l}\text { Schizocosa ocreata immature male } 1 \text {, de novo tran- } \\
\text { scriptome assembly }\end{array}$ & FASTA files (.fa) & $\begin{array}{l}\text { NCBI TSA: https://identifiers.org/ncbi/insdc:GIZNO } \\
0000000 \text { [47] }\end{array}$ \\
\hline Data set 3 & $\begin{array}{l}\text { Schizocosa ocreata immature male } 2 \text {, de novo tran- } \\
\text { scriptome assembly }\end{array}$ & FASTA files (.fa) & $\begin{array}{l}\text { NCBI TSA: https://identifiers.org/ncbi/insdc:GIZSO } \\
0000000 \text { [48] }\end{array}$ \\
\hline Data set 4 & $\begin{array}{l}\text { Schizocosa ocreata immature male } 3 \text {, de novo tran- } \\
\text { scriptome assembly }\end{array}$ & FASTA files (.fa) & $\begin{array}{l}\text { NCBI TSA: https://identifiers.org/ncbi/insdc:GIZTO } \\
0000000 \text { [49] }\end{array}$ \\
\hline Data set 5 & $\begin{array}{l}\text { Schizocosa ocreata immature female } 2 \text {, de novo } \\
\text { transcriptome assembly }\end{array}$ & FASTA files (.fa) & $\begin{array}{l}\text { NCBI TSA: https://identifiers.org/ncbi/insdc:GIZMO } \\
0000000 \text { [50] }\end{array}$ \\
\hline Data set 6 & $\begin{array}{l}\text { Schizocosa ocreata immature female } 3 \text {, de novo } \\
\text { transcriptome assembly }\end{array}$ & FASTA files (.fa) & $\begin{array}{l}\text { NCBI TSA: https://identifiers.org/ncbi/insdc:GIZRO } \\
0000000 \text { [51] }\end{array}$ \\
\hline Data set 7 & $\begin{array}{l}\text { Schizocosa ocreata mature male } 2 \text {, de novo transcrip- } \\
\text { tome assembly }\end{array}$ & FASTA files (.fa) & $\begin{array}{l}\text { NCBI TSA: https://identifiers.org/ncbi/insdc:GIZPO } \\
0000000 \text { [52] }\end{array}$ \\
\hline Data set 8 & $\begin{array}{l}\text { Schizocosa ocreata mature male } 3 \text {, de novo transcrip- } \\
\text { tome assembly }\end{array}$ & FASTA files (.fa) & $\begin{array}{l}\text { NCBI TSA: https://identifiers.org/ncbi/insdc:GIZWO } \\
0000000 \text { [53] }\end{array}$ \\
\hline Data set 9 & $\begin{array}{l}\text { Schizocosa ocreata mature female } 1 \text {, de novo tran- } \\
\text { scriptome assembly }\end{array}$ & FASTA files (.fa) & $\begin{array}{l}\text { NCBI TSA: https://identifiers.org/ncbi/insdc:GIZOO } \\
0000000 \text { [54] }\end{array}$ \\
\hline Data set 10 & $\begin{array}{l}\text { Schizocosa ocreata mature female } 2 \text {, de novo tran- } \\
\text { scriptome assembly }\end{array}$ & FASTA files (.fa) & $\begin{array}{l}\text { NCBI TSA: https://identifiers.org/ncbi/insdc:GIZU0 } \\
0000000 \text { [55] }\end{array}$ \\
\hline Data set 11 & $\begin{array}{l}\text { Schizocosa ocreata mature female } 3 \text {, de novo tran- } \\
\text { scriptome assembly }\end{array}$ & FASTA files (.fa) & $\begin{array}{l}\text { NCBI TSA: https://identifiers.org/ncbi/insdc:GIZVO } \\
0000000 \text { [56] }\end{array}$ \\
\hline Data set 12 & Schizocosa ocreata consensus coding sequences & FASTA file (.fa) & $\begin{array}{l}\text { NCBI TSA: https://identifiers.org/ncbi/insdc:GIZQ0 } \\
0000000 \text { [57] }\end{array}$ \\
\hline Data file 2 & Transcriptome assembly statistics & PDF file (.pdf) & NCBI GEO https://identifiers.org/geo:GSE168766 [46] \\
\hline Data file 3 & Gene annotations & Tabular text file (.txt) & NCBI GEO https://identifiers.org/geo:GSE168766 [46] \\
\hline Data file 4 & Gene expression values & Tabular text file (.txt) & NCBI GEO https://identifiers.org/geo:GSE168766 [46] \\
\hline Data file 5 & Differential expression values & MS excel file (.xIsx) & NCBI GEO https://identifiers.org/geo:GSE168766 [46] \\
\hline Data file 6 & $\begin{array}{l}\text { Transcriptome flow (TFLOW): de novo transcriptome } \\
\text { analysis pipeline }\end{array}$ & Zip archive (.zip) & Zenodo https://doi.org/10.5281/zenodo.3817474 [58] \\
\hline Data file 7 & Gene clustering analysis script & Python script (.py) & Zenodo https://doi.org/10.5281/zenodo.4330738 [59] \\
\hline Data file 8 & Differential expression analysis script & R script (.R) & Zenodo https://doi.org/10.5281/zenodo.4330738 [59] \\
\hline
\end{tabular}

in well-studied model organisms [19-25]. The data presented here are valuable in laying necessary groundwork for broad comparative functional genomics of sex differences in brain and behavior across arthropods.

\section{Data description}

mRNA was isolated from brain samples of immature (Imm; subadult) and mature (Mat; adult) male and female Schizocosa ocreata, collected in Lancaster County, Nebraska (see detailed methods: NCBI Gene Expression Omnibus [GEO] Series accession number GSE168766). Illumina paired-end sequencing was performed with libraries generated from mRNA derived from individual brain samples, with three replicates for each sex/ stage (Data set 1; NCBI SRA: SRP302932). Sequence reads were processed to remove index and low-quality sequences; quality assessments are provided (Data file 1; Table 1; GEO GSE168766).

Transcriptome assembly was performed for each sample using Trinity followed by CAP3 [26, 27]. A consensus transcriptome was assembled combining all individual assemblies using CAP3. Transcriptome quality was evaluated on individual sample and consensus transcriptome assemblies based on the number of conserved protein coding genes identified from the Core Eukaryotic Genes Mapping Approach (CEGMA) and Benchmarking sets of Universal Single-Copy Orthologs (BUSCO) Arthropod databases (Data file 2; GEO GSE168766) [28-30]. Both CEGMA and BUSCO alignments used the Basic Local Alignment Search (BLAST) utility with default threshold E-value of $1 \mathrm{e}-20$ [31]. In the consensus assembly, 99\% of CEGMA and $95 \%$ of BUSCO genes were identified, 
demonstrating a high assembly quality. To facilitate this workflow, the Transcriptome-Flow (TFLOW) pipeline was developed (Python 2.7; Zenodo; Data file 6). Each individual assembly was filtered to remove contaminant sequences and uploaded to the NCBI Transcriptome Shotgun Assembly (TSA) database (Data sets 2-11; Table 1).

Putative protein coding sequences from the consensus assembly were extracted using TransDecoder v5.3.0 [32]. Coding sequences were annotated using Trinonate [33], aligning coding sequence (CDS) and predicted protein sequences against several databases, including Uniprot (October 2018), NCBI nr, and the Flybase Drosophila melanogaster v6.23 draft genome (Data file 3; GEO GSE168766). These alignments were used for identification of Protein Family (Pfam) domains, Gene Ontology (GO), and Kyoto Encyclopedia of Genes and Genomes (KEGG) identifiers [34-39].

Annotated coding sequences were clustered into genes based on sequence similarity determined by an all-vsall BLASTn analysis, with software archived on Zenodo (Data file 7). Sequences identified as contaminants were removed and consensus CDS sequences were uploaded to the TSA database (Data set 12: TSA GIZQ00000000). For analysis of differential expression, reads were aligned to consensus CDS sequences and assigned to gene clusters, with expression estimated as read counts per gene (Data file 4; GEO GSE168766). Read alignment was performed using Burrows-Wheeler Aligner (BWA-MEM, version 0.6.1-r104) [40, 41]. A linear model was fit with the glmLRT function in edgeR (version 3.1.2) using default (trimmed mean of $M$ values, TMM) normalization [42-44]. Likelihood ratio tests were constructed with comparisons between: (1) immature vs mature adult data within sex; (2) all immature vs all adult data from both sexes; (3) male vs. female data at each stage; and (4) all male vs all female data from both stages. The calculated $\log$ fold-change ( $\log \mathrm{FC}), \log$ counts-per-million $(\log C P M)$, Likelihood-ratio (LR), p-value, and false discovery rate (FDR) adjusted p-value are reported (Data file 5; GEO GSE168766). The R-script has been archived on Zenodo (Data file 8).

\section{Limitations}

Following read processing and quality assessment, two libraries (immature female 1 and adult male 1) were excluded from further analysis due to low sequence coverage. This limits the power to detect differential expression in the corresponding comparisons. The quality of the transcriptomes could be improved by coupling these data with long-read sequencing data in future work. Since the completion of this study, the CEGMA annotation database has been discontinued. The TFLOW software package was developed in the Python 2.7 programming language which is no longer actively supported. Archival versions of Python 2.7 may be utilized to execute TFLOW, or conversion of this software to a currently-supported version of Python can be performed using a python version-update package.

\section{Abbreviations \\ CEGMA: Core Eukaryotic Genes Mapping Approach; BUSCO: Benchmarking sets of Universal Single-Copy Orthologs; BLAST: Basic Local Alignment Search Tool; BWA: Burrows-Wheeler Aligner; CDS: Coding sequence; FDR: False discovery rate; GO: Gene Ontology; GEO: Gene Expression Omnibus; Imm: Immature; KEGG: Kyoto Encyclopedia of Genes and Genomes; logCPM: Log counts-per-million; logFC: Log fold-change; LR: Likelihood-ratio; Mat: Mature; mRNA: Messenger RNA; NCBI: National Center for Biotechnology Information; Pfam: Protein family; TMM: Trimmed mean of M-value; RNA-seq: RNA sequenc- ing; TSA: Transcriptome Shotgun Assembly; SRA: Sequence Read Archive.}

\section{Acknowledgements}

We thank Professor Gary Tyson in Computer Science at Florida State University for supporting DS

\section{Authors' contributions}

The project was conceived by MNA. JD made the Illumina sequencing libraries with brain tissue collected by MR utilizing a protocol designed by EH. DS and PLC developed the software that was utilized and performed the computational analyses. CAC created the clustering analysis. DS, PLC, RMG, and MNA performed data interpretation and manuscript writing. All authors provided intellectual feedback, and reviewed, manuscript submission. All authors read and approved the final manuscript.

\section{Funding}

This study was supported by research start-up funds from the Florida State College of Medicine (awarded to MNA) and National Science Foundation (NSF) Grant 1751296 (awarded to RMG).

\section{Availability of data and materials}

The data described in this Data note can be freely and openly accessed on the NCBI SRA database under accession number SRP302932 (https://ident ifiers.org/ncbi/insdc.sra:SRP302932); the NCBI TSA database under BioProject accession number PRJNA694130 (https://identifiers.org/bioproject:PRJNA 694130; full accession list in Table 1); and the NCBI GEO database under accession number GSE168766 (https://identifiers.org/geo:GSE168766) [45-57]. The computational pipeline utilized for transcriptome assembly and quality assessment is available through Zenodo under DOI: https://doi.org/10.5281/zenodo. 3817475 [58]. The scripts utilized for clustering and differential expression analysis are available through Zenodo under DOl: https://doi.org/10.5281/ zenodo.4330738 [59]. Please see Table 1 and references [45-59] for details and links to the data.

\section{Declarations}

Ethics approval and consent to participate

Not applicable.

\section{Consent for publication \\ Not applicable.}

\section{Competing interests}

The authors declare no competing interests. 


\section{Author details}

'Biomedical Sciences Department, College of Medicine, Florida State University, Tallahassee, FL 32306, USA. ${ }^{2}$ Department of Biological Sciences, University of Southern California, Los Angeles, CA 90089, USA. ${ }^{3}$ School of Biological Sciences, University of Nebraska-Lincoln, Lincoln, NE 68588, USA. ${ }^{4}$ Department of Biological Sciences, Auburn University, Auburn, AL 36849, USA. ${ }^{5}$ Present Address: Department of Molecular Genetics and Microbiology, Genetics Institute, University of Florida, Gainesville, FL 32610, USA

Received: 22 March 2021 Accepted: 9 June 2021

Published online: 23 June 2021

\section{References}

1. Schneider J, Fromhage L. Monogynous mating strategies in spiders. In: Kappeler P, editor. Animal behaviour: evolution and mechanisms. Berlin: Springer; 2010. p. 441-64. https://doi.org/10.1007/978-3-642-02624-9_15.

2. Herberstein ME, Wignall AE, Hebets EA, Schneider JM. Dangerous mating systems: signal complexity, signal content and neural capacity in spiders. Neurosci Biobehav Rev. 2014;46(Pt 4):509-18. https://doi.org/10.1016/j. neubiorev.2014.07.018.

3. Shamble PS, Wilgers DJ, Swoboda KA, Hebets EA. Courtship effort is a better predictor of mating success than ornamentation for male wolf spiders. Behav Ecol. 2009;20:1242-51. https://doi.org/10.1093/beheco/ arp116.

4. Mouginot $P$, Uhl G, Fromhage L. Evolution of external female genital mutilation: why do males harm their mates? R Soc Open Sci. 2017;4: 171195. https://doi.org/10.1098/rsos.171195.

5. Řezáč M. The spider Harpactea sadistica: co-evolution of traumatic insemination and complex female genital morphology in spiders. Proc R Soc B Biol Sci. 2009;276:2697-701. https://doi.org/10.1098/rspb.2009. 0104.

6. Kamimura Y. Correlated evolutionary changes in Drosophila female genitalia reduce the possible infection risk caused by male copulatory wounding. Behav Ecol Sociobiol. 2012;66:1107-14. https://doi.org/10. 1007/s00265-012-1361-0.

7. Kamimura Y. Twin intromittent organs of Drosophila for traumatic insemination. Biol Lett. 2007;3:401-4. https://doi.org/10.1098/rsbl.2007.0192

8. $\mathrm{Ng}$ CS, Kopp A. Sex combs are important for male mating success in Drosophila melanogaster. Behav Genet. 2008;38:195-201. https://doi.org/ 10.1007/s10519-008-9190-7.

9. Spieth HT. Mating behaviour within the genus Drosophila (Diptera). Bull Am Mus Nat Hist. 1952;99:395-474.

10. Cobb M, Burnet B, Connolly K. The structure of courtship in the Drosophila melanogaster species sub-group. Behaviour. 1986:97:182-212.

11. Reinhardt K, Anthes N, Lange R. Copulatory wounding and traumatic insemination. Cold Spring Harb Perspect Biol. 2015. https://doi.org/10 1101/cshperspect.a017582.

12. Masly JP, Kamimura Y. Asymmetric mismatch in strain-specific genital morphology causes increased harm to Drosophila females. Evolution. 2014;68:2401-11. https://doi.org/10.1111/evo.12436.

13. Tanaka K, Barmina O, Massey JH, Kopp A. Convergent evolution of sex-specific leg ornaments in Drosophilidae-from cells to structures. BioRxiv. 2019. https://doi.org/10.1101/786764.

14. Kopp A. Drosophila sex combs as a model of evolutionary innovations. Evol Dev. 2011;13:504-22. https://doi.org/10.1111/j.1525-142X.2011. 00507.x.

15. Greenspan RJ, Ferveur JF. Courtship in Drosophila. Annu Rev Genet. 2000;34:205-32. https://doi.org/10.1146/annurev.genet.34.1.205.

16. Garb JE, Sharma PP, Ayoub NA. Recent progress and prospects for advancing arachnid genomics. Curr Opin Insect Sci. 2018;25:51-7. https://doi.org/10.1016/j.cois.2017.11.005.

17. Pomerantz AF, Hoy MA, Kawahara AY. Molecular characterization and evolutionary insights into potential sex-determination genes in the western orchard predatory mite Metaseiulus occidentalis (Chelicerata: Arachnida: Acari: Phytoseiidae). J Biomol Struct Dyn. 2015;33:1239-53. https://doi. org/10.1080/07391102.2014.941402.

18. Meibers HE, Finch G, Gregg RT, Glenn S, Assani KD, Jennings EC, et al. Sex- and developmental-specific transcriptomic analyses of the Antarctic mite, Alaskozetes antarcticus, reveal transcriptional shifts underlying oribatid mite reproduction. Polar Biol. 2018. https://doi.org/10.1007/ s00300-018-2427-X

19. Moskalik B, Uetz GW. Experience with chemotactile cues indicating female feeding history impacts male courtship investment in the wolf spider Schizocosa ocreata. Behav Ecol Sociobiol. 2011;65:2175-81. https:// doi.org/10.1007/s00265-011-1225-z.

20. McClintock WJ, Uetz GW. Female choice and pre-existing bias: visual cues during courtship in two Schizocosawolf spiders (Araneae: Lycosidae). Anim Behav. 1996;52:167-81. https://doi.org/10.1006/anbe.1996.0162.

21. McLean CJ, Garwood RJ, Brassey CA. Sexual dimorphism in the Arachnid orders. PeerJ. 2018;6:e5751. https://doi.org/10.7717/peerj.5751.

22. Walker SE, Rypstra AL. Sexual dimorphism in trophic morphology and feeding behavior of wolf spiders (Araneae: Lycosidae) as a result of differences in reproductive roles. Can J Zool. 2002;80:679-88. https://doi.org/ 10.1139/z02-037.

23. Hebets EA, Wesson J, Shamble PS. Diet influences mate choice selectivity in adult female wolf spiders. Anim Behav. 2008;76:355-63. https://doi.org/ 10.1016/j.anbehav.2007.12.021.

24. Hebets EA, Uetz GW. Leg ornamentation and the efficacy of courtship display in four species of wolf spider (Araneae: Lycosidae). Behav Ecol Sociobiol. 2000;47:280-6. https://doi.org/10.1007/s002650050667.

25. Wilgers DJ, Hebets EA. Age-related female mating decisions are condition dependent in wolf spiders. Behav Ecol Sociobiol. 2012;66:29-38. https:// doi.org/10.1007/s00265-011-1248-5.

26. Grabherr MG, Haas BJ, Yassour M, Levin JZ, Thompson DA, Amit I, et al. Full-length transcriptome assembly from RNA-Seq data without a reference genome. Nat Biotechnol. 2011;29:644-52. https://doi.org/10.1038/ nbt.1883.

27. Huang X, Madan A. CAP3: a DNA sequence assembly program. Genome Res. 1999;9:868-77. https://doi.org/10.1101/gr.9.9.868.

28. Parra G, Bradnam K, Korf I. CEGMA: a pipeline to accurately annotate core genes in eukaryotic genomes. Bioinformatics. 2007;23:1061-7. https:// doi.org/10.1093/bioinformatics/btm071.

29. Parra G, Bradnam K, Ning Z, Keane T, Korf I. Assessing the gene space in draft genomes. Nucleic Acids Res. 2009;37:289-97. https://doi.org/10. 1093/nar/gkn916.

30. Seppey M, Manni M, Zdobnov EM. BUSCO: assessing genome assembly and annotation completeness. Methods Mol Biol. 2019;1962:227-45. https://doi.org/10.1007/978-1-4939-9173-0_14.

31. Altschul SF, Gish W, Miller W, Myers EW, Lipman DJ. Basic local alignment search tool. J Mol Biol. 1990;215(410):403. https://doi.org/10.1006/jmbi. 1990.9999.

32. Haas BJ, Papanicolaou A, Yassour M, Grabherr M, Blood PD, Bowden J, et al. De novo transcript sequence reconstruction from RNA-seq using the Trinity platform for reference generation and analysis. Nat Protoc. 2013;8:1494-512. https://doi.org/10.1038/nprot.2013.084.

33. Bryant DM, Johnson K, DiTommaso T, Tickle T, Couger MB, Payzin-Dogru $D$, et al. A tissue-mapped axolotl de novo transcriptome enables identification of limb regeneration factors. Cell Rep. 2017;18:762-76. https://doi. org/10.1016/j.celrep.2016.12.063.

34. El-Gebali S, Mistry J, Bateman A, Eddy SR, Luciani A, Potter SC, et al. The Pfam protein families database in 2019. Nucleic Acids Res. 2019;47:D42732. https://doi.org/10.1093/nar/gky995.

35. Ashburner M, Ball CA, Blake JA, Botstein D, Butler H, Michael Cherry J, et al. Gene ontology: tool for the unification of biology. Nat Genet. 2000;25:25-9. https://doi.org/10.1038/75556.

36. The Gene Ontology Consortium. The gene ontology resource: 20 years and still going strong. Nucleic Acids Res. 2019;47:D330-8. https://doi.org/ 10.1093/nar/gky1055.

37. Kanehisa M, Goto S. KEGG: kyoto encyclopedia of genes and genomes. Nucleic Acids Res. 2000;28:27-30. https://doi.org/10.1093/nar/28.1.27.

38. Kanehisa M. Toward understanding the origin and evolution of cellular organisms. Protein Sci. 2019;28:1947-51. https://doi.org/10.1002/pro. 3715.

39. Kanehisa M, Furumichi M, Sato Y, Ishiguro-Watanabe M, Tanabe M. KEGG: integrating viruses and cellular organisms. Nucleic Acids Res. 2020. https://doi.org/10.1093/nar/gkaa970.

40. Li H. Aligning sequence reads, clone sequences and assembly contigs with BWA-MEM. arXiv preprint. arxiv:1303.3997. 2013. 
41. Li H, Durbin R. Fast and accurate long-read alignment with BurrowsWheeler transform. Bioinformatics. 2010;26:589-95. https://doi.org/10. 1093/bioinformatics/btp698.

42. Robinson MD, McCarthy DJ, Smyth GK. edgeR: a Bioconductor package for differential expression analysis of digital gene expression data. Bioinformatics. 2010;26:139-40. https://doi.org/10.1093/bioinformatics/ btp616.

43. McCarthy DJ, Chen Y, Smyth GK. Differential expression analysis of multifactor RNA-Seq experiments with respect to biological variation. Nucleic Acids Res. 2012. https://doi.org/10.1093/nar/gks042.

44. Chen Y, Lun ATL, Smyth GK. Differential expression analysis of complex RNA-seq experiments using edgeR. Stat Anal Next Gener Seq Data. 2014. https://doi.org/10.1007/978-3-319-07212-8_3.

45. Stribling D, Chang PL, Dalton JE, Conow CA, Rosenthal M, Hebets E, Graze RM, Arbeitman MN. NCBI Sequence Read Archive. 2021. https://ident ifiers.org/ncbi/insdc.sra:SRP302932.

46. Stribling D, Chang PL, Dalton JE, Conow CA, Rosenthal M, Hebets E, Graze RM, Arbeitman MN. The brain transcriptome of the wolf spider, Schizocosa ocreata. NCBI Gene Expression Omnibus. 2021. https://identifiers. org/geo:GSE168766.

47. Stribling D, Chang PL, Dalton JE, Conow CA, Graze RM, Arbeitman MN. TSA: Schizocosa ocreata isolate immature male, transcriptome shotgun assembly. NCBI Transcriptome Shotgun Assembly Sequence Database. 2021. https://identifiers.org/ncbi/insdc:GIZN00000000.

48. Stribling D, Chang PL, Dalton JE, Conow CA, Graze RM, Arbeitman MN. TSA: Schizocosa ocreata isolate immature male, transcriptome shotgun assembly. NCBI Transcriptome Shotgun Assembly Sequence Database. 2021. https://identifiers.org/ncbi/insdc:GIZS00000000.

49. Stribling D, Chang PL, Dalton JE, Conow CA, Graze RM, Arbeitman MN. TSA: Schizocosa ocreata isolate immature male, transcriptome shotgun assembly. NCBI Transcriptome Shotgun Assembly Sequence Database. 2021. https://identifiers.org/ncbi/insdc:GIZT00000000.

50. Stribling D, Chang PL, Dalton JE, Conow CA, Graze RM, Arbeitman MN TSA: Schizocosa ocreata isolate immature female, transcriptome shotgun assembly. NCBI Transcriptome Shotgun Assembly Sequence Database. 2021. https://identifiers.org/ncbi/insdc:GIZM00000000.

51. Stribling D, Chang PL, Dalton JE, Conow CA, Graze RM, Arbeitman MN. TSA: Schizocosa ocreata isolate immature female, transcriptome shotgun assembly. NCBI Transcriptome Shotgun Assembly Sequence Database. 2021. https://identifiers.org/ncbi/insdc:GIZR00000000.

52. Stribling D, Chang PL, Dalton JE, Conow CA, Graze RM, Arbeitman MN TSA: Schizocosa ocreata isolate mature male, transcriptome shotgun assembly. NCBI Transcriptome Shotgun Assembly Sequence Database. 2021. https://identifiers.org/ncbi/insdc:GIZP00000000.

53. Stribling D, Chang PL, Dalton JE, Conow CA, Graze RM, Arbeitman MN. TSA: Schizocosa ocreata isolate mature male, transcriptome shotgun assembly. NCBI Transcriptome Shotgun Assembly Sequence Database. 2021. https://identifiers.org/ncbi/insdc:GIZW00000000.

54. Stribling D, Chang PL, Dalton JE, Conow CA, Graze RM, Arbeitman MN. TSA: Schizocosa ocreata isolate mature female, transcriptome shotgun assembly. NCBI Transcriptome Shotgun Assembly Sequence Database. 2021. https://identifiers.org/ncbi/insdc:GlZO00000000.

55. Stribling D, Chang PL, Dalton JE, Conow CA, Graze RM, Arbeitman MN. TSA: Schizocosa ocreata isolate mature female, transcriptome shotgun assembly. NCBI Transcriptome Shotgun Assembly Sequence Database. 2021. https://identifiers.org/ncbi/insdc:GIZU00000000.

56. Stribling D, Chang PL, Dalton JE, Conow CA, Graze RM, Arbeitman MN. TSA: Schizocosa ocreata isolate mature female, transcriptome shotgun assembly. NCBI Transcriptome Shotgun Assembly Sequence Database. 2021. https://identifiers.org/ncbi/insdc:GIZV00000000.

57. Stribling D, Chang PL, Dalton JE, Conow CA, Graze RM, Arbeitman MN. TSA: Schizocosa ocreata, transcriptome shotgun assembly. NCBI Transcriptome Shotgun Assembly Sequence Database. 2021. https://identifiers. org/ncbi/insdc:GIZQ00000000.

58. D Stribling. Transcriptome-FLOW (TFLOW): transcriptome assembly pipeline. Zenodo. 2015. https://doi.org/10.5281/zenodo.3817474

59. Stribling D, Chang PL. Schizocosa ocreata gene clustering and differential expression analysis scripts (version v1.1). Zenodo. 2020. https://doi.org/ 10.5281/zenodo.4330738.

\section{Publisher's Note}

Springer Nature remains neutral with regard to jurisdictional claims in published maps and institutional affiliations.
Ready to submit your research? Choose BMC and benefit from:

- fast, convenient online submission

- thorough peer review by experienced researchers in your field

- rapid publication on acceptance

- support for research data, including large and complex data types

- gold Open Access which fosters wider collaboration and increased citations

- maximum visibility for your research: over $100 \mathrm{M}$ website views per year

At BMC, research is always in progress.

Learn more biomedcentral.com/submissions 